\title{
A CULTURA ORGANIZACIONAL SEGUNDO A PERCEPÇÃO DE CHEFES DE SERVIÇOS DE ENFERMAGEM DE HOSPITAIS DE DIFERENTES GRUPOS ÉTNICOS
}

\author{
ORGANIZATIONAL CULTURE ACCORDING TO HEAD NURSES PERCEPTION
}

\section{LA CULTURA ORGANIZATIVA SEGÚN LA PERCEPCIÓN DE JEFAS DE SERVICIOS DE ENFERMERÍA DE HOSPITALES DE DIFERENTES GRUPOS ÉTNICOS}

\author{
Victoria Secaf* \\ Paulina Kurcgant**
}

\begin{abstract}
Secaf v, Kurcgant P. A cultura organizacional segundo a percepção de chefes de serviços de enfermagem de hospitais de diferentes grupos étnicos. Rev Esc Enferm USP 2001; 35(3):282-6.
\end{abstract}

\section{RESUMO}

As chefes do Serviço de Enfermagem que atuam e atuaram em cinco Hospitais, criados e mantidos por comunidades de imigrantes de diferentes países, na cidade de São Paulo, expressam neste estudo, a influência da cultura da organização em seu desempenho no cargo. O relato oral de cada uma delas em entrevista, a partir de um roteiro, evidenciou a identidade, a trajetória profissional, a permanência na instituição, bem como o relacionamento com as demais chefias e com os elementos do grupo étnico.

PALAVRAS-CHAVE: Enfermeiras Chefe. Cultura organizacional. Grupo étnico.

\begin{abstract}
The Nursing Service Managers from five Hospitals, all created and mantained by immigration communities, in São Paulo city, express in this study the influence of the culture of the organization on their job performance. The verbal report of each of them in na interview, starting from a template, has evidenced the identify the professional life, the remaining on the institution as well the relationship with other managers and with the individuals of the ethnic group.
\end{abstract}

KEYWORDS: Nursing Supervisory. Organizational Culture. Ethnic groups.

\section{RESUMEN}

Las jefas del servicio de enfermeria que actúan y actuaron en cinco hospitales, creados y mantenidos por comunidades de inmigrantes de diferentes países, en la ciudad de São Paulo, expresan, en este estudio, la influencia de la cultura de la organización en su desempeño en el cargo. El relato oral de cada una de ellas en entrevista, partiendo de un cuestionario, evidenció la identidad, la trayectoria profesional, la permanencia en la institución, así como la relación con las demás jefaturas y con los elementos del grupo étnico.

PALABRAS-CLAVE: Enfermeras jefas. Cultura organizacional. Grupos étnicos.

* Enfermeira. Doutor em Enfermagem, Docente da Escola de Enfermagem da Universidade de Silo Paulo.

** Enfermeira. Doutor em Enfermagem, Livre-Docente em Enfermagem. Professor Titular de Enfermagem da Universidade de São Paulo.pkurcg@usp.br 


\section{CONSIDERAÇÕES INICIAIS}

Nas últimas décadas, no Brasil, ocorreram alterações significativas no que se refere a organização do setor hospitalar e é sabido que a assistência à saúde obedeceu inicialmente ao modelo existente em Portugal e a primeira Santa Casa de Misericórdia inaugurada foi a de Santos, no Estado de São Paulo, em 1543.

Posteriormente, foram criados hospitais de iniciativa governamental ou de entidades privadas, de sociedades de medicina de grupo e também surgiram instituições mantidas por comunidades de imigrantes em várias capitais brasileiras ${ }^{(1)}$.

Além de passar por inúmeras mudanças nas práticas científicas e tecnológicas, a enfermagem, como profissão, também avançou muito no desempenho dos diferentes processos de trabalho.

No que se refere ao processo de cuidar e gerenciar considera-se que é de muita importância a inserção da atual Lei do Exercício Profissional e a obrigatoriedade legal de enfermeira na chefia do Serviço de Enfermagem (SE) dos hospitais, além daquelas que administram unidades e prestam assistência de enfermagem direta aos pacientes.

A chefia do SE por enfermeira, mesmo decretada por legislação antiga e específica (Lei n $\left.{ }^{\circ} 775 / 49\right)$ não era cumprida em muitos hospitais de Estados avançados no setor saúde, como o de São Paulo(2)

No estudo as autoras procuraram reconstruir, historicamente, o processo de criação e manutenção de cinco hospitais ligados a comunidades de imigrantes, na cidade de São Paulo com o objetivo de resgatar determinadas características desses hospitais quanto às influências do grupo de origem(1).

Passada uma década desde o trabalho realizado em 1988, pode-se constatar que os hospitais, de comunidades de imigrantes, continuaram com registro de suas trajetórias e contribuições significativas no atendimento à saúde da população brasileira.

É de salientar o crescimento quanti/qualitativo do pessoal de enfermagem e a participação efetiva das enfermeiras na assistência e no gerenciamento dos Serviços de Enfermagem. Foi nessa trajetória que houve em alguns hospitais a mudança da denominação do cargo hierárquico de Chefe do Serviço de Enfermagem para Gerente de Enfermagem.

Evidentemente, do ponto de vista histórico, aquele estudo foi satisfatório, e revelou que seria válido evidenciar ainda o aspecto dinâmico, isto é, o histórico temporal e sua influência na cultura da organização segundo a perspectiva das chefes dos Serviços de Enfermagem desses hospitais.

Estudos sobre fatores que influenciam as formas e estilos de gerenciamento de enfermagem em hospitais brasileiros nas duas últimas décadas têm sido foco de publicações.
Entretanto, até o momento não tem sido devidamente pesquisado o vivencial das chefes de enfermagem na história de cada hospital.

Naturalmente, foi considerado válido, também, conhecer as características que os profissionais apresentam ou deveriam apresentar quando o hospital, "locus" de seu viver profissional, numa instituição criada e mantida por determinado grupo étnico.

\section{O REFERENCIAL TEMÁTICO}

Para alguns antropólogos, sociólogos, psicólogos e administradores que estudam a cultura organizacional, o fato de instituições ou empresas terem sido criadas e mantidas por grupos étnicos, guardam como elementos integrantes da cultura organizacional, valores, crenças, símbolos, rituais, ritos e mitos próprios da cultura do grupo étnico que lhes deu origem.

O termo "cultura da empresa" é a suposta capacidade de um dado grupo (os gerentes), através da utilização de ritos, cerimônias, símbolos e mitos apropriados, suscitar, reforçar ou modificar valores, atitudes e crenças consideradas "eficazes" à empresa (3)

Como estudioso de cultura organizacional, considera que efetivamente os símbolos, os mitos, as crenças, ou seja, todos os elementos da superestrutura não existem de forma independente, mas suas existências só são possiveis através de suas relações com elementos correspondentes da infraestrutura, ou seja, é preciso, primeiro, que isso tudo seja experimentado e vivido de fato. Somente após essa experiência vivida é que podem ser delineados os contornos dos elementos que favorecem uma relação positiva entre o individuo, os demais indivíduos e a organização enquanto estrutura social. Para tanto, são importantes os elementos: proximidade concreta e vínculos afetivos com os dirigentes; ausência quase total de privilégios exclusivos; ausência quase total de sinais de distanciamento ou de diferença de "status"; comportamento habitual exemplarmente generoso, justo e igualitário por parte dos dirigentes; efetiva participação e cooperação - todos são ouvidos, há diálogos, autonomia e a realização de cerimônias que estão em harmonia com a vida de trabalho e a vida social da empresa sendo a continuação do convivio existente dentro da mesma.

As atitudes, as crenças, os simbolos, vêm depois, eles são alimentados por todos estes elementos concretamente materiais.

O mesmo autor atribui grande importância ao papel dos fundadores, no processo de moldar os padrões culturais da organização, considerando que os primeiros líderes, ao desenvolverem formas próprias de resolver problemas da organização, acabam por imprimir a sua visão de mundo aos demais membros 
como também a sua visão a respeito da missão que a organização deve desempenhar na sociedade.

Considera também, para o reconhecimento da cultura de uma dada organização, que devem ser analisados o processo de socialização dos novos membros, as respostas aos incidentes críticos que ocorreram durante a vida da organização e as crenças, valores e convicções dos criadores ou portadores da cultura organizacional.

Segundo estudioso do assunto as manifestações simbólicas e as práticas sociais das organizações encontram, numa determinada ideologia, a chave que lhes confere articulações e coerência(4).

Alguns antropólogos tenderam a conceber os padrões culturais não como um molde que produziria condutas estritamente idênticas, mas como regras de um jogo, isto é, uma estrutura que permitisse atribuir significado a certas ações e em função da qual fossem jogadas infinitas partidas (5)

Já para alguns administradores a criação e manutenção de uma cultura organizacional, devem ser firmadas as concepções, normas e valores que devem ser comunicados aos membros da organização de uma forma tangivel (6).

Esta forma tangivel da cultura organizacional são os ritos, os rituais, os mitos, as estórias, os gestos, os artefatos.

É importante considerar que a existência de uma continuidade cultural não tem nada a ver com o caráter evolutivo da organização de uma sociedade ou de uma instituição.

Assim, os fatos e os comportamentos importam, na medida do que significam e esses significados variam conforme a cultura. Isto quer dizer que a cultura é um contexto de significados exclusivos e cambiáveis(7).

A cultura organizacional, embora muito considerada na área empresarial, não tem sido devidamente focalizada nos estudos que tratam do gerenciamento em saúde.

Seria o presente trabalho uma possibilidade de despertar a atenção das enfermeiras para uma reflexão sobre o tema?

\section{OBJETIVOS}

Foram estabelecidos como objetivos:

- $\quad$ Conhecer a trajetória profissional de chefes dos Serviços de Enfermagem de hospitais de comunidades de imigrantes na cidade de São Paulo.

- Resgatar a influência do grupo étnico na dinâmica organizacional desses hospitais do ponto de vista das enfermeiras que foram (ou ainda são) chefes do Serviço de Enfermagem.

\section{O PERCURSO METODOLÓGICO}

A História Oral foi a escolha metodológica inicial, por possibilitar conhecer cada sujeito da pesquisa e mesmo o grupo, no caso, chefes de SE de hospitais ligados à comunidades de imigrantes.

A história oral é um instrumento de coleta de dados como também o são o depoimento e a biografia, entre outros (8).

A oralidade é o elemento comum entre eles e o depoimento pode ser o escolhido quando o pesquisador pretende evidenciar dados específicos que o sujeito tem conhecimento.

Assim, o relato oral foi adotado por permitir dar destaque à percepção que as enfermeiras-chefes dos Serviços de Enfermagem dos hospitais ligados às comunidades de imigrantes têm quanto ao seu desempenho no cargo de chefia.

Ainda, o relato oral foi a técnica escolhida para a coleta de dados, visto que se pretende evidenciar a condição histórico-temporal vivenciada pelas chefes do $\mathrm{SE}$ e a influência do grupo étnico nas relações interpessoais e de poder.

Para a realização do estudo, os cinco hospitais da cidade de São Paulo e seus respectivos grupos étnicos foram Hospital São Joaquim da Real e Benemérita Beneficência Portuguesa (português), Oswaldo Cruz (alemão), Sírio-Libânes (árabe), Albert Einstein (israelita) e Nipo Brasileiro (japonês). Neles estavam inseridas as chefes do SE sendo que algumas permanecem, até a presente data e outras, por diferentes motivos, não mais exercem o referido cargo nessas instituições.

Dessa forma, o critério de seleção de sujeitos adotado pelas autoras foi que seriam entrevistadas somente as enfermeiras que eram chefes do SE, na época da realização do estudo anterior (1988).

Inicialmente, cada sujeito do estudo foi contatado por carta ou telefone, para agendar a data e horário da entrevista que teve por base um roteiro (Anexo I).

O roteiro da entrevista, antecedido do devido consentimento informado e assinado, com a garantia do anonimato, foi adotado pelas autoras/ entrevistadoras e a sequência dos tópicos, determinada pela própria depoente.

Para manutenção do anonimato foi registrada a identificação das depoentes como El, E2, E3, E4 e E5, sendo a letra E (de Enfermeira) seguida do número correspondente a cada hospital onde cada uma estava atuando na época do estudo anterior.

Cada relato foi gravado, transcrito e lido várias vezes pelas autoras a fim de serem resgatados os significados essenciais para explicitação dos objetivos do estudo. 
$\mathrm{Na}$ análise dos achados foram transcritos, em negrito, trechos das falas das depoentes.

\section{RESULTADOS}

Pela análise dos achados foi constatado que todas as entrevistadas tiveram experiências anteriores como enfermeiras, em outros hospitais, com exceção de uma delas que "graduou-se em 1976, recebeu a franquia e foi lá, para se oferecer para trabalhar. Acabou ficando no pré-operatório". (E4)

Das cinco chefes de enfermagem entrevistadas, três delas passaram por processo seletivo, na instituição, para admissão como enfermeira (E1,E3,E4). Uma delas (El) resgata esse momento na fala: "um hospital que dava treinamento e aceitava recém-formado". As enfermeiras El e E4 antes de assumirem a chefia do SE referem ter sido designadas para supervisão ou de uma área ou de um turno de trabalho.

As outras duas (E2, E5) foram selecionadas, especificamente para o cargo de chefes do SE, sendo que uma delas (E2) refere "o processo seletivo foi através de firma externa... foi de forma muito negativa". A seguir, esta mesma enfermeira foi convidada para uma entrevista com elementos da entidade mantenedora quando, segundo ela: "entrei para o cargo.."

Outra refere "... fui convidada pelo médico que trabalhava também no (hospital X) que vinha a ser o Superintendente aqui. Vim para cá a convite. Para montar o serviço de enfermagem. Enfim o hospital que, na época, estava em fase de construção e permaneci na chefia de enfermagem". (E5)

Ainda quanto à identificação, mesmo antes de sua admissão ou durante a permanência nos hospitais ligados à comunidades de imigrantes, estas enfermeiras participaram de cursos de pós-graduação (strito e lato sensu) sendo que duas delas completaram o doutorado.

Em relação às respostas sobre o tempo de permanência no cargo, cabe ressaltar que duas enfermeiras continuam sendo chefes do SE no final de 2000, desenvolvendo a enfermagem durante a sua trajetória profissional nas instituições há mais de 20 anos. (E1,E3). A outra permaneceu no cargo como chefe do SE desde a inauguração do hospital em 1988 até 1996 e continua atuando como assessora junto à administração geral. (E5). Teve também, uma enfermeira que permaneceu como chefe do SE durante 20 anos e 4 meses, desligando-se no momento da aposentadoria. (E2).

Diferenciando-se das demais chefes entrevistadas, uma delas, já desligada, permaneceu na instituição por 13 anos, dos quais 8 deles, como Chefe do $\mathrm{SE}$ e relata que seu desligamento do hospital foi decorrente de mudança na politica administrativa da instituição. (E4).
No que se refere ao relacionamento das chefes do SE com o grupo étnico representado pelos integrantes dos órgãos deliberativos dos hospitais, foi considerado, por elas, como uma experiência muito positiva. As entrevistadas depõem que o desenvolvimento, aprimoramento e transformações ocorridas nas instituições são decorrentes do gerenciamento e das propostas assistenciais de enfermagem e em grande parte, do entendimento consensual entre cada uma delas e os representantes do grupo étnico. Assim, em algumas falas revelam: "Com - grupo étnico sempre foi de muita confiança", uma relação muito honesta" (E4). Resgata-se também, nas falas, que estas enfermeiras chefes, sentiram-se privilegiadas em terem participado déssas vivências, "Eu ainda acho que os melhores hospitais para se trabalhar são do grupo étnico". (E2). Outra explicitou: "os próprios diretores tinham interesse em saber como iam as coisas. Eu tenho orgulho de ter trabalhado lá; fazer o melhor". (E4).

Um outro aspecto constatado é que os representantes do grupo étnico, seja como participante do grupo deliberativo ou como membro do primeiro escalão executivo, constituem presença marcante, detentora de poder e, geralmente permanecem, por muitos anos, na instituição e no referido cargo.

Merece destaque ainda o fato de não ter sido relatado, por quatro delas, a não influência da etnia do candidato, no processo seletivo de enfermeiras ou médicos.

Entretanto, uma das chefes entrevistadas relatou que devido à necessidade de comunicação com os pacientes, na lingua materna, havia preferência em selecionar profissionais do mesmo grupo étnico.

$O$ último aspecto que foi considerado nas entrevistas, está relacionado com o resgate do cotidiano, do vivencial de ser chefe do SE num hospital ligado a grupo étnico. Neste aspecto foram explicitadas como mostram as falas a seguir, as características do profissional como da instituição.

Os depoimentos seguintes reiteram isto.

"A gente trabalha, diretamente, ligada com a administração... como é muito familiar eu acho que muita coisa fica presa em pessoas, não é decisão de departamento, por exemplo, é decisão pessoal". (E1).

Outra refere que "foi gratificante porque a administração deu total condições de atuação; de plena liberdade de ação; responsabilidade com autoridade. Tive todo apoio em todos os caminhos". (E2).

E a entrevistada (E3) considera que "é um hospital de projeção da comunidade". "Tem que ser uma administradora flexivel". A E4 diz: "Os próprios diretores tinham (como grupo) interesse em saber como iam as coisas". 


\section{CONSIDERAÇÕES FINAIS}

Os achados neste trabalho vêm ao encontro do exposto pelos estudiosos de cultura organizacional evidenciando uma relação positiva entre a chefe do $\mathrm{SE}$ e o(s) representante(s) do grupo étnico onde atua no referente à identificação de valores, crenças, concepções e significados.

Outra evidência que referenda a relação positiva entre os elementos acima citados é o tempo de permanência de cada uma delas no cargo e na respectiva instituição. A aceitação das chefes, por parte da instituição e o fato das chefes aceitarem as condições materiais e principalmente relacionais próprias da instituição são referendadas pelo convivio duradouro e expressas por palavras elogiosas.

Fica evidente ainda, nos depoimentos, o sentimento de orgulho das enfermeiras por terem sido ou permanecer ainda como integrantes desses hospitais, bem como a identificação com os valores e crenças do correspondente grupo étnico.

Apesar da comprovação da capacitação técnica e gerencial de cada uma das chefes, sem dúvida, a identificação com os valores e crenças do correspondente grupo étnico é a característica mais evidenciada pelas enfermeiras deste estudo.

Esta pesquisa permite considerar que enfermeiras, Chefes de Serviços de Enfermagem, percebem a identificação com os valores e crenças da cultura organizacional como um elemento facilitador do desempenho no cargo.

\section{REFERÊNCIAS BIBLIOGRÁFICAS}

(1) Secaf V, Kurcgant P, Sawaia BB. Hospitais criados por comunidades de imigrantes na cidade de São Paulo. Rev Baiana Enferm 1988; 4(2):116-83

(2) Secaf V. Quem chefia os serviços de enfermagem nos hospitais do Estado de São Paulo, Mundo da Saúde 1979; 3(19):88-90.

(3) Chanlat JF, coordenador. O individuo na organização: dimensões esquecidas. São Paulo: Atlas; 1992 .

(4) Srour RH. Poder, cultura e ética nas organizações. Rio de Janeiro: Campus; 1998. A cultura nas organizações; cap.6, p. $169-80$.

(5) 7urhan E. Cultura e ideologia. Rev Ci Soc 1984; 27 (1):

(6) Beyer J, Trice H. How an organization's rites reveal it's culture. [s.1.]: Organizational Dynamics; 1986.

(7) Fleury, MTL, Fisher, RM. Cultura e poder nas organizações. São Paulo: Atlas; 1996. $<<<<<<$ enegro, AT. História oral e memória: cultura popular revisitada. São Paulo: Contexto; 1992.

\section{ANEXO I}

\section{Roteiro da Entrevista}

A sua história na Instituição...

Identificação, processo seletivo, admissão

Permanência (quanto tempo, como: . Enfermeira .

. Chefe do SE

Capacitação Profissional: - anterior

- durante

O relacionamento com:

- Chefias (técnica, administrativa)

- Elementos do grupo étnico

- Enfermeiras

O seu cotidiano:

Como é ser chefe num hospital ligado a grupo étnico (quais as características para este trabalho). 